\title{
Chapter

\section{An updated checklist of fauna of Binsar wildlife sanctuary of Uttarakhand (Western Himalaya), India}

\author{
Manoj Kumar Arya*, Aarti Badoni, \\ Aman Verma and Ambika Tiruwa
}

Insect Biodiversity Laboratory, Department of Zoology, D.S.B. Campus, Kumaun University, Nainital 263002, India

\begin{abstract}
The present checklist on faunal composition is a compilation of published records on faunal diversity of mammals, Aves and insects mainly between the years 1998-2021 from the Binsar Wildlife Sanctuary in State Uttarakhand, India. The sanctuary is known for its wide range of mammals, birds and insect species. The list comprised 16 species of mammals belonging to five orders under nine families, 173 species of birds belonging to 12 orders under 45 families and 121 species of insects belonging to eight orders under 36 families. It is intended that the checklist would help in obtaining a holistic view of faunal diversity, mainly mammals, birds and insects so that their status could be monitored and maintained at various levels in the sanctuary. The sanctuary is low profiled in terms of biodiversity conservation and scientific management, and still unexplored or under-explored in relation to the faunal diversity. Therefore, the findings reported in the present study promisingly emphasize the ongoing threats to the biological diversity of the Binsar wildlife sanctuary of Uttarakhand (Western Himalaya), India which needs an immediate attention in order to conserve the residing faunal species.
\end{abstract}

\section{Keywords Diversity, Fauna, Himalaya, Insects}

$\triangle$ Manoj Kumar Arya, Email: dr.manojkumar19@rediffmail.com ( ${ }^{*}$ Corresponding author) (C) 2021 | Agro Environ Media | Agriculture and Environmental Science Academy, Haridwar, India 


\section{Introduction}

The hill state of Uttarakhand located in Western Himalayan Region covers an area of 53,483 sq. km stretched between $28^{\circ} 43^{\prime}$ to $31^{\circ} 28^{\prime}$ North Latitudes and $77^{\circ} 34^{\prime}$ to $81^{\circ} 03^{\prime}$ East Longitudes. Based on the administrative attributes, there are 13 districts covered in Garhwal and Kumaon Hills (Arya and Verma, 2020). With approximately $64.79 \%$ of total land under forest cover, area of about $9,885 \mathrm{sq} . \mathrm{km}$ $(18.48 \%)$ of the state has been insulated by the creation and management of protected area network for in situ conservation of biodiversity and fragile ecosystems (Rodgers and Panwar, 1988). The existing network of protected areas in the Himalayan Region covers about $6 \%$ of the entire range, and most of them suffer from human and biotic pressures and lack of sufficient management inputs as well as adequate conservation plans (Rawal and Dhar, 2001). The protected areas located in the Indian Himalayan Region especially those which are low profiled ones hold immense potential to enhance the components of floral and faunal representativeness, integrity and human sustenance in the region (Rawal and Dhar, 2001). At present there are six national parks, seven wildlife sanctuaries and four conservation reserves in the state Uttarakhand.

Binsar Wildlife Sanctuary (BWLS) represents one of the oldest protected landscapes in the Kumaon region, and it is a natural habitat for several flora and fauna. The sanctuary recently received the attention of government and non-government organizations, and is being developed as a hot tourist destination in the calm and pristine environment of the Kumaon Himalaya. Protected areas of Indian Himalayan Region offer unique habitats for studying the diversity of different groups of mammals, birds, insects and other faunal groups. Many scientific records regarding the flora, wildlife, birds and various aspects of ecological and environmental studies have been published by workers of different organizations of international and national repute from the BWLS (Ilyas, 1998; Ilyas, 1999; Khan et al., 2000; Sultana and Khan, 2000; Ilyas and Khan, 2001, 2004, 2005; Islam and Rahmani, 2004; Singh and Khushwah, 2011; Kala and Majila, 2013; Kala and Kothari, 2013; Uttarakhand Forest Department, 2014, Bhalla et al., 2015; BirdLife International, 2021; Mohan and Sondhi, 2014, 2015, 2017; Shahabuddin et al., 2017; Bhalla et al., 2020; Lepage, 2021). Similarly, studies on different aspects of biodiversity have been carried out in the BWLS of Himalaya viz. natural resource utilization (Majila and Kala, 2010), ecosystem functions (Majila et al., 2005), forest conservation (Rawat et al., 2013), community-based ecotourism (Bhalla et al., 2017), floristic analysis (Khan and Arya, 2017) and human-wildlife conflicts (Kala and Kothari, 2013). Recently, studies on the ecology and behavior of insects have been carried out by Ghosh et al. (2011, 2018), Arya et al. (2016a, b), Arya et al. (2017), Tamta (2017), Arya et al. (2018a, b), Arya et al. (2019) and Arya et al. (2020) in different locations of the BWLS. However, comprehensive attempts to understand the faunal diversity including mammals, birds and insects are still lacking from the sanctuary.

A detailed checklist representing faunal repository is essential, and is required for the scientific and planned management of species in the BWLS. Therefore, the purpose of this study was to examine and evaluate the reported faunal diversity in the BWLS through considering published literature and 
authentic accessible reports in order to establish a reliable biodiversity data source for future conservation in the protected area. The present study also aims to generate information for conservation authorities regarding the development and management of the sanctuary.

\section{Binsar Wildlife Sanctuary}

It is stretched between $29^{\circ} 39^{\prime}$ to $29^{\circ} 44^{\prime}$ North Latitudes and $79^{\circ} 41^{\prime}$ to $79^{\circ} 49^{\prime}$ E Longitudes is located in districts Almora and Bageshwar of Uttarakhand at an altitude of 1200 to 2500 meters above sea level in Kumaon Himalaya (Figure 1). Binsar is a fascinating spot that offers a majestic glimpse of the snowcapped Indian Himalayan peaks namely-Nanda Devi, Trishul and Panchachuli, presenting a unique experience to its visitors. With the geographical area of $47.67 \mathrm{sq}$. km, the sanctuary has core zone $\left(4 \mathrm{~km}^{2}\right)$ and buffer zone $\left(43.67 \mathrm{~km}^{2}\right)$. No human activity is allowed in the Core Zone (Restricted Zone). Prior to India's Independence, it was notified as "Protected Forest" in 1893 and later upgraded as "Reserve" Forest in 1897. After Independence in 1947, its status was revived to "Wildlife Sanctuary" by the Government of India in the year 1988.

\section{Zoning within BWLS}

Zone permits focus on specific areas in order to accommodate different management needs as per the requirement of the protected landscape. Within BWLS, different areas catered different needs and demands from diversified sections of the society (Bhalla et al., 2015). Therefore, to meet these requirements zonation of the sanctuary was framed legally (Binsar Management Plan, 2000-2010) in a most scientific, sustainable and logical manner by the regional forest department in consultation with the villagers and concerned stakeholders as follows:

- Core zone: It is actually a mini core zone $\left(4 \mathrm{~km}^{2}\right)$ that comprises forest area of strategic importance, mainly different oak tree species along with its rich under storey biodiversity. Extraction of forest produce in form of right and concessions is seldom allowed.
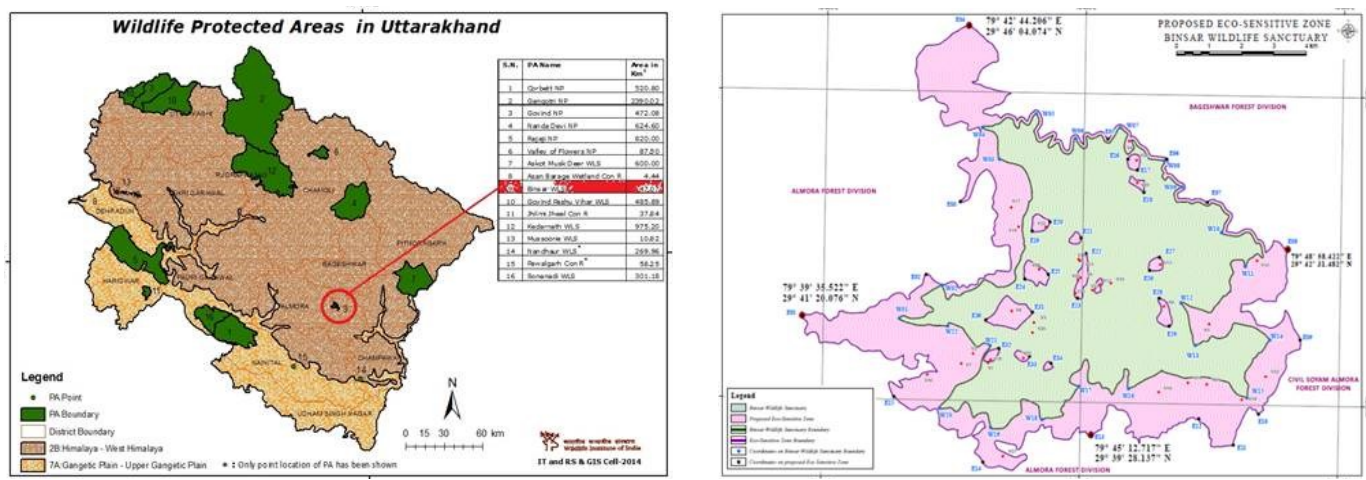

Figure 1. Location of Binsar Wildlife Sanctuary in Uttarakhand (Source: WII, 2014). 
- Tourism zone: It comprises of the motor road passing through Binsar compartments, which terminates at the forest rest house. From here a nature trail is made upto Jhandi Dhar (Zero-point), highest point of BWLS, from where a magnificent view of mighty Himalayas is seen. All resort accommodations and four villages lay within this zone, that provide as tourist attractions.

- Buffer zone: Rest of the areas not covered in above two zones fall under the Buffer zone. These areas are open for regulated grazing and address the rights and concessions requirement of villagers. At present there are two entry gates for visitors into BWLS, one at Ayarpani, and other at Dhaulchina, which lay within this zone along with two villages.

- Eco-development zone: Covers the area up to $5 \mathrm{~km}$ from the boundary of BWLS. It comprises of a several villages and few resorts for visitor accommodations.

\section{Climate}

The climatic conditions prevailing in the BWLS range from temperate to sub-arctic. Winters are very cold and the region receives heavy snowfall during December-February. The mean monthly temperature ranges from $2.2^{\circ} \mathrm{C}$ to $15.5^{\circ} \mathrm{C}$ during winter and from $17.20^{\circ} \mathrm{C}$ to $26.6^{\circ} \mathrm{C}$ during summer, with the average rainfall of more than $1200 \mathrm{~mm}$ (Sharma et al., 1999). The mean maximum temperature varies from $14.30^{\circ} \mathrm{C}$ (January) to $31.73^{\circ} \mathrm{C}$ (June) and the mean minimum temperature fluctuates from $0.2^{\circ} \mathrm{C}$ (January) to $12.30^{\circ} \mathrm{C}$ (August). During the coldest months of January and February, the area receives heavy dew at night. Snowfall usually starts in the middle of December and continues till the end of February. The monsoon generally begins after mid June. Sometimes it may start around late May or mid-July and last till the end of September. The heaviest rainfall usually occurs in July and August (Kala and Majila, 2013). Relative humidity varies along aspects and types of vegetation in the BWLS. The average relative humidity varies on a monthly basis from $36 \%$ to $76 \%$ in the Chir Pine forest during April and August, respectively. In the Oak forest, the relative humidity varies between 49\% in April and 87\% in August (Majila, 1992 and Majila et al., 2005).

\section{Geology, geomorphology and soil}

The diverse climatic, geographical, and topographical conditions have shaped the diverse forest vegetation and wildlife species in BWLS. On an average, the soil is very rich in organic matter that contains a high proportion of plant nutrients. The slopes vary from steep to very steep. The terrain has been shaped by the action of running water. Throughout the sanctuary, the terrain is hilly and characterized by deep ravines, crevices and elevated ridges (Bhalla et al., 2015). Geologically, the sanctuary falls under the Inner Lesser Himalayan zone, which mainly comprises unfossiliferous sedimentary rocks of various ages, that is, from Precambrian to Paleozoic (Valdia, 1976). The rocks are predominantly made up of the Korl group of Paleozoic age, which are composed of granite, granodiorite, graphite, schist, shale-quartzite, and quartzite (Gansser, 1964). 


\section{Flora of Binsar}

Binsar represents the characteristic floral element of moist temperate type of forest (Saxena and Singh, 1982). BWLS is known for its wide variety of flora including 40 species of trees followed by 26 shrubs, 50 herbs, 19 grasses and six ferns (Ilyas, 1998). The forested hilltops and slopes in the sanctuary are covered with Chir Pine (Pinus roxburghii), Banj Oak (Quercus leucotrichophora), and Rhododendron (Rhododendron arboreum) as pure stands or as mixed forests. Pure pine forests are found at the altitude ranging between $1600 \mathrm{~m}$ to $1900 \mathrm{~m}$, while mixed forests of pine and oak are distributed between 1900 $\mathrm{m}$ to $2100 \mathrm{~m}$ altitudes. Pure oak and mixed-oak forests (Quercus leucotrichophora and Quercus floribunda) are present between $2100 \mathrm{~m}$ to $2400 \mathrm{~m}$ altitudinal range in the sanctuary (Majila and Kala, 2010). In the more recent years, Rawat et al. (2013) reported a total of 147 plant species in BWLS of which 90 species were herbs, 20 were shrubs and 27 were trees. Among them, there are 12 species with edible fruits, three species are timber yielding, 26 are good fuel and fodder, eight have sacred values and 46 are medicinally important.

\section{Data collection, compilation and evaluation}

Secondary data on recorded faunal species of mammals, birds and insects was obtained from the available published literature (Ilyas, 1998; Ilyas, 1999; Khan et al., 2000; Sultana and Khan, 2000; Ilyas and Khan, 2001, 2004; Islam and Rahmani, 2004; Ilyas and Khan, 2005; Majila and Kala, 2010; ZSI, 2010; Singh and Khushwah, 2011; Ghosh et al., 2011; Kala and Majila, 2013; Kala and Kothari, 2013; Rawat et al., 2013; Mohan and Sondhi, 2014; Uttarakhand Forest Department, 2014; Bhalla et al., 2015; Mohan and Sondhi, 2015; Arya et al., 2016a, b; BirdLife International, 2021; Bhalla et al., 2017; Khan and Arya, 2017; Mohan and Sondhi, 2017; Arya et al., 2017; Tamta, 2017; Ghosh et al., 2018; Arya et al., 2018a, b; Arya et al., 2019; Arya et al., 2020; Shahsbuddin et al., 2017; Arya and Verma, 2020; Bhalla et al., 2020 and Lepage, 2021). The species in the checklist were catalogued alphabetically into orders, families, genera and all the species were listed with their accepted names as such with correct spellings. However, an updated classification and latest scientific names of bird species were followed as per Grimmett et al. (2011).

\section{Taxonomic composition of faunal species in BWLS}

BWLS is known for its wide range of mammals, birds and insect species. The compilation of data from available literature resulted in 16 species of mammals belonging to five orders under nine families, 173 species of birds belonging to 12 orders under 45 families and 121 species of insects belonging to eight orders under 36 families as reported from BWLS of Uttarakhand.

\section{Mammalian diversity}

India hosts rich diversity of flora and fauna. The total 410 species of mammals in India comprises about $8.9 \%$ of all known mammal species worldwide (Nameer, 1998). BWLS is home to 16 high altitude species of mammals (Table 1) including Common Leopard (Panthera pardus), Jungle Cat (Felis chaus), 
Table 1. Mammal species composition of Binsar Wildlife Sanctuary.

\begin{tabular}{|c|c|c|c|}
\hline Order/ Family & Common Name & Scientific Name & Status: IW(P)A and IUCN \\
\hline $\begin{array}{l}\text { CARNIVORA/ } \\
\text { Falidae }\end{array}$ & Common Leopard & $\begin{array}{l}\text { Panthera pardus } \\
\text { (Linnaeus) }\end{array}$ & $\begin{array}{l}\text { IW(P)A: Schedule I, Part I } \\
\text { VU (Nationally), DD } \\
\text { (Globally) }\end{array}$ \\
\hline $\begin{array}{l}\text { CARNIVORA/ } \\
\text { Falidae }\end{array}$ & Jungle Cat & Felis chaus Schreber & $\begin{array}{l}\text { IW(P)A: Schedule II } \\
\text { LRnt (Nationally), DD } \\
\text { (Globally) }\end{array}$ \\
\hline $\begin{array}{l}\text { CARNIVORA/ } \\
\text { Ursidae }\end{array}$ & $\begin{array}{l}\text { Himalayan Black } \\
\text { Bears }\end{array}$ & (Selenarctos thibetans) & $\begin{array}{l}\text { VU (Nationally), DD } \\
\text { (Globally) }\end{array}$ \\
\hline $\begin{array}{l}\text { CARNIVORA/ } \\
\text { Canidae }\end{array}$ & Jackals & $\begin{array}{l}\text { Canis aureus Linnae- } \\
\text { us }\end{array}$ & $\begin{array}{l}\text { IW(P)A: Schedule I, Part I } \\
\text { LRnt (Nationally), DD } \\
\text { (Globally) }\end{array}$ \\
\hline $\begin{array}{l}\text { CARNIVORA/ } \\
\text { Canidae }\end{array}$ & Red Fox & $\begin{array}{l}\text { Vulpes vulpes } \\
\text { (Linnaeus) }\end{array}$ & $\begin{array}{l}\text { IW(P)A: Schedule II, Part II } \\
\text { LRlc (Nationally), DD } \\
\text { (Globally) }\end{array}$ \\
\hline $\begin{array}{l}\text { CARNIVORA/ } \\
\text { Mustelidae }\end{array}$ & $\begin{array}{l}\text { Yellow-throated Mar- } \\
\text { tin }\end{array}$ & $\begin{array}{l}\text { Martes flavigula } \\
\text { (Baddaert) }\end{array}$ & $\begin{array}{l}\text { IW(P)A: Schedule II, Part II } \\
\text { LRnt (Nationally), DD } \\
\text { (Globally) }\end{array}$ \\
\hline $\begin{array}{l}\text { ARTIODAC- } \\
\text { TYLA/ Bovidae }\end{array}$ & Goral & $\begin{array}{l}\text { Nemorhaedus goral } \\
\text { (Hardwicke) }\end{array}$ & IUCN: LR/nt \\
\hline $\begin{array}{l}\text { ARTIODAC- } \\
\text { TYLA/ Bovidae }\end{array}$ & Serow & $\begin{array}{l}\text { Nemorhaedus suma- } \\
\text { trensis ((Bechstein) }\end{array}$ & $\begin{array}{l}\text { IW(P)A: Schedule I, Part I } \\
\text { VU (Nationally), DD } \\
\text { (Globally) }\end{array}$ \\
\hline $\begin{array}{l}\text { ARTIODAC- } \\
\text { TYLA/ Cervidae }\end{array}$ & Barking Deer & $\begin{array}{l}\text { Muntiacus muntjak } \\
\text { (Zimmermann) }\end{array}$ & $\begin{array}{l}\text { IW(P)A: Schedule III } \\
\text { LRlc (Nationally), DD } \\
\text { (Globally) }\end{array}$ \\
\hline $\begin{array}{l}\text { ARTIODAC- } \\
\text { TYLA/ Suidae }\end{array}$ & Wild Boars & Sus scrofa Linnaeus & $\begin{array}{l}\text { IW(P)A: Schedule III } \\
\text { LRlc (Nationally), DD } \\
\text { (Globally) }\end{array}$ \\
\hline $\begin{array}{l}\text { PRIMATES/ Cer- } \\
\text { copithecidae }\end{array}$ & Rhesus Macaques & $\begin{array}{l}\text { Macaca mulatta } \\
\text { (Zimmermann) }\end{array}$ & $\begin{array}{l}\text { IW(P)A: Schedule II } \\
\text { LRlc (Nationally), DD } \\
\text { (Globally) }\end{array}$ \\
\hline $\begin{array}{l}\text { PRIMATES/ Cer- } \\
\text { copithecidae }\end{array}$ & Common Langurs & (Presbytus entellus) & $\begin{array}{l}\text { IW(P)A: Schedule II } \\
\text { LRlc (Nationally), DD } \\
\text { (Globally) }\end{array}$ \\
\hline $\begin{array}{l}\text { RODENTIA/ } \\
\text { Sciuridae }\end{array}$ & Giant Flying Squirrel & $\begin{array}{l}\text { Petaurista petaurista } \\
\text { (Pallas) }\end{array}$ & Not Known \\
\hline $\begin{array}{l}\text { RODENTIA/ } \\
\text { Sciuridae }\end{array}$ & $\begin{array}{l}\text { Kashmir Flying } \\
\text { Squirrel }\end{array}$ & Hylopetes fimbriatus & Not Known \\
\hline $\begin{array}{l}\text { RODENTIA/ } \\
\text { Hystricidae }\end{array}$ & $\begin{array}{l}\text { Indian Crested Por- } \\
\text { cupine }\end{array}$ & Hystrix indica Kerr & $\begin{array}{l}\text { IW(P)A: Schedule IV } \\
\text { LRlc (Nationaly), DD } \\
\text { (Globaly) }\end{array}$ \\
\hline $\begin{array}{l}\text { LAGOMORPHA/ } \\
\text { Leporidae }\end{array}$ & Black-napped Hare & $\begin{array}{l}\text { Lepus nigricollis } \mathrm{Cu}- \\
\text { vier }\end{array}$ & DD (Nationally \& Globally) \\
\hline
\end{tabular}

(Abbreviations used: IW(P)A= Indian Wildlife (Protection) Act, VU= Vulnerable, LR/nt or LRnt= Lower Risk near threatened, LRlc = Lower Risk least concern, DD= Data Deficient, Schedule I, II, III, IV of Indian Wildlife (Protection) Act, 1972) 
Himalayan Black Bear (Selenarctos thibetans), Jackal (Canis aureus) and red fox (Vulpes vulpes). Major Ungulate species includes Gorals (Nemorhaedus goral), Barking Deer (Muntiacus muntjak), Serows (Nemorhaedus sumatrensis) and Wild Boars (Sus scrofa). Other mammal species in the sanctuary are Rhesus Macaques (Macaca mulatta), Common Langurs (Presbytus entellus), Giant Flying Squirrel (Petaurista petaurista), Kashmir Flying Squirrel (Hylopetes fimbriatus), Indian Crested Porcupine (Hystrix indica) and Black-napped Hare (Lepus nigricollis). Of the total reported mammals, 11 species are legally protected under different schedules of the Indian Wildlife (Protection) Act, 1972 (Table 1).

\section{Avian diversity}

Uttarakhand is home to 14 important Bird Areas (Islam and Rahmani, 2004) that are important and priority sites for conservation (Mohan and Sondhi, 2017). BWLS is rich in avian diversity, it has been declared as an important bird area IBA (A3) by BirdLife International. The sanctuary harbors 173 species of birds under 12 orders belonging to 45 families (Table 2) and some of the common species of birds reported in the sanctuary named as, Koklass Pheasants (Pucrasia macrolopha), Kaleej Phaesants (Lophura leucomelana), Hill Partridges (Arborophilla torqueola), Great Barbets (Megalaima virens), Himalayan Griffons (Gyps himalayensis), Black Francolins (Fracolinus francolinus), Mountain Hawk Eagle (Nisaetus nipalensis), Lammergeiers (Gypaetus barbatus),White-throated Tit (Aegithalous niveogularis), Red -billed Blue Magpies (Urocissa erythrorhyncha) and Yellow-Billed Magpies (Urocissa flavirostris). Total number of species of bird's orders and their families compiled from the BWLS is presented in Figure 2. As per the IUCN Red List of Threatened Species, five species are near threatened, one species is vulnerable, one species is endangered, one species is critically endangered and 161 species are least concerned (Table 2).

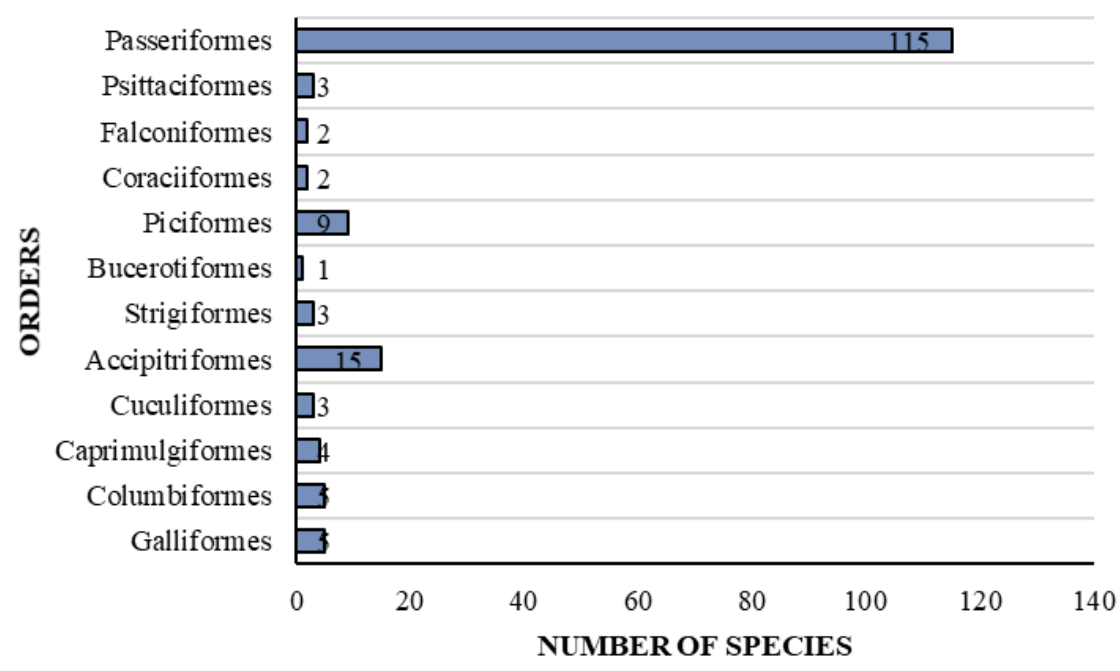

Figure 2. Number of species of bird's orders and their families reported from the BWLS. 
Table 2. Avian faunal species composition of Binsar Wildlife Sanctuary.

\begin{tabular}{|c|c|c|c|}
\hline ORDER/Family & Common Name & Scientific Name & $\begin{array}{l}\text { Status: } \\
\text { IUCN }\end{array}$ \\
\hline \multirow{15}{*}{$\begin{array}{l}\text { ACCIPITRIFORMES / } \\
\text { Accipitridae }\end{array}$} & Lammergeier & Gypaetus barbatus & NT \\
\hline & Egyptian Vulture & Neophron percnopterus & E \\
\hline & Crested Serpent Eagle & Spilornis cheela & $\mathrm{LC}$ \\
\hline & Red-headed Vulture & Sarcogyps calvus & $\mathrm{CE}$ \\
\hline & Himalayan Vulture & Gyps himalayensis & NT \\
\hline & Mountain Hawk Eagle & Nisaetus nipalensis & $\mathrm{LC}$ \\
\hline & Black Eagle & Ictinaetus malayensis & $\mathrm{LC}$ \\
\hline & Tawny Eagle & Aquila rapax & $\mathrm{V}$ \\
\hline & Golden Eagle & Aquila chrysaetos & $\mathrm{LC}$ \\
\hline & Bonelli's Eagle & Aquila fasciata & $\mathrm{LC}$ \\
\hline & Shikra & Accipiter badius & $\mathrm{LC}$ \\
\hline & Eurasian Sparrowhawk & Accipiter nisus & LC \\
\hline & Lesser Fish Eagle & Icthyophaga humilis & NT \\
\hline & Black Kite & Milvus migrans & LC \\
\hline & Upland Buzzard & Buteo hemilasius & LC \\
\hline $\begin{array}{l}\text { BUCEROTIFORMES/ } \\
\text { Upupidae }\end{array}$ & Common Hoopoe & Upupa epops & $\mathrm{LC}$ \\
\hline CAPRIMULGI- & Grey Nightjar & Caprimulgus indicus & $\mathrm{LC}$ \\
\hline $\begin{array}{l}\text { FORMES / } \\
\text { Caprimulgidae }\end{array}$ & Large-tailed Nightjar & Caprimulgus macrurus & $\mathrm{LC}$ \\
\hline CAPRIMULGI- & White-throated Needletail & Hirundapus caudacutus & $\mathrm{LC}$ \\
\hline FORMES/ Apodidae & Fork-tailed Swift & Apus pacificus & LC \\
\hline \multirow{5}{*}{$\begin{array}{l}\text { COLUMBIFORMES/ } \\
\text { Columbidae }\end{array}$} & Wood Pigeon & Columba palumbus & LC \\
\hline & Oriental Turtle Dove & Streptopelia orientalis & LC \\
\hline & Spotted-necked Dove & Stigmatopelia chinensis & LC \\
\hline & Yellow-legged Green Pigeon & Treron phoenicopterus & LC \\
\hline & Wedge-tailed Green Pigeon & Treron sphenurus & $\mathrm{LC}$ \\
\hline \multirow{2}{*}{$\begin{array}{l}\text { CORACIIFORMES/ } \\
\text { Alcedinidae }\end{array}$} & Common Kingfisher & Alcedo atthis & LC \\
\hline & White-throated Kingfisher & Halcyon smyrnensis & $\mathrm{LC}$ \\
\hline \multirow{3}{*}{$\begin{array}{l}\text { CUCULIFORMES/ } \\
\text { Cuculidae }\end{array}$} & Large Hawk Cuckoo & Hierococcyx sparverioides & $\mathrm{LC}$ \\
\hline & Indian Cuckoo & Cuculus micropterus & $\mathrm{LC}$ \\
\hline & Common Cuckoo & Cuculus canorus & LC \\
\hline \multirow{2}{*}{$\begin{array}{l}\text { FALCONIFORMES/ } \\
\text { Falconidae }\end{array}$} & Common Kestrel & Falco tinnunculus & LC \\
\hline & Peregrine Falcon & Falco peregrinus & $\mathrm{LC}$ \\
\hline \multirow{5}{*}{$\begin{array}{l}\text { GALLIFORMES/ } \\
\text { Phasianidae }\end{array}$} & Common Hill Partridge & Arborophila torqueola & LC \\
\hline & Chukar Partridge & Alectoris chukar & LC \\
\hline & Black Francolin & Francolinus francolinus & LC \\
\hline & Kalij Pheasant & Lophura leucomelanos & LC \\
\hline & Koklass Pheasant & Pucrasia macrolopha & LC \\
\hline PASSERIFORMES/ & Long-tailed Minivet & Pericrocotus ethologus & $\mathrm{LC}$ \\
\hline Campephagidae & Black-winged Cuckooshrike & Lalage melaschistos & $\mathrm{LC}$ \\
\hline
\end{tabular}


Table 2. Continued...

\begin{tabular}{|c|c|c|c|}
\hline ORDER/Family & Common Name & Scientific Name & $\begin{array}{l}\text { Status } \\
\text { IUCN }\end{array}$ \\
\hline $\begin{array}{l}\text { PASSERIFORMES/ } \\
\text { Vireonidae }\end{array}$ & Himalayan Shrike-babbler & Pteruthius ripleyi & $\mathrm{LC}$ \\
\hline \multirow{3}{*}{$\begin{array}{l}\text { PASSERIFORMES/ } \\
\text { Oriolidae }\end{array}$} & Maroon Oriole & Oriolus traillii & $\mathrm{LC}$ \\
\hline & Indian Golden Oriole & Oriolus kundoo & LC \\
\hline & Black-naped Oriole & Oriolus chinensis & $\mathrm{LC}$ \\
\hline $\begin{array}{l}\text { PASSERIFORMES/ } \\
\text { Vangidae }\end{array}$ & Bar-winged Flycatcher-shrike & Hemipus picatus & $\mathrm{LC}$ \\
\hline \multirow{4}{*}{$\begin{array}{l}\text { PASSERIFORMES/ } \\
\text { Dicruridae }\end{array}$} & Black Drongo & Dicrurus macrocercus & $\mathrm{LC}$ \\
\hline & Ashy Drongo & Dicrurus leucophaeus & $\mathrm{LC}$ \\
\hline & Bronzed Drongo & Dicrurus aeneus & $\mathrm{LC}$ \\
\hline & Hair-crested Drongo & Dicrurus hottentottus & LC \\
\hline $\begin{array}{l}\text { PASSERIFORMES/ } \\
\text { Rhipiduridae }\end{array}$ & White-throated Fantail & Rhipidura albicollis & $\mathrm{LC}$ \\
\hline \multirow{7}{*}{$\begin{array}{l}\text { PASSERIFORMES/ } \\
\text { Corvidae }\end{array}$} & Grey Treepie & Dendrocitta formosae & $\mathrm{LC}$ \\
\hline & Red-billed Blue Magpie & Urocissa erythroryncha & $\mathrm{LC}$ \\
\hline & Yellow-billed Magpie & Urocissa flavirostris & LC \\
\hline & Eurasian Jay & Garrulus glandarius & LC \\
\hline & Black-headed Jay & Garrulus lanceolatus & LC \\
\hline & House Crow & Corvus splendens & $\mathrm{LC}$ \\
\hline & Large-billed Crow & Corvus macrorhynchos & LC \\
\hline $\begin{array}{l}\text { PASSERIFORMES/ } \\
\text { Dicaeidae }\end{array}$ & Fire breasted Flowerpecker & Dicaeum ignipectus & $\mathrm{LC}$ \\
\hline \multirow{2}{*}{$\begin{array}{l}\text { PASSERIFORMES/ } \\
\text { Nectariniidae }\end{array}$} & Black-throated Sunbird & Aethopyga saturata & LC \\
\hline & Green-tailed Sunbird & Aethopyga nipalensis & $\mathrm{LC}$ \\
\hline \multirow{3}{*}{$\begin{array}{l}\text { PASSERIFORMES/ } \\
\text { Prunellidae }\end{array}$} & Alpine Accentor & Prunella collaris & $\mathrm{LC}$ \\
\hline & Robin Accentor & Prunella rubeculoides & $\mathrm{LC}$ \\
\hline & Rufous-breasted Accentor & Prunella strophiata & $\mathrm{LC}$ \\
\hline \multirow{2}{*}{$\begin{array}{l}\text { PASSERIFORMES/ } \\
\text { Estrildidae }\end{array}$} & Indian Silverbill & Euodice malabarica & $\mathrm{LC}$ \\
\hline & Scaly-breasted Munia & Lonchura punctulata & $\mathrm{LC}$ \\
\hline \multirow{3}{*}{$\begin{array}{l}\text { PASSERIFORMES/ } \\
\text { Passeridae }\end{array}$} & House Sparrow & Passer domesticus & $\mathrm{LC}$ \\
\hline & Russet Sparrow & Passer cinnamomeus & $\mathrm{LC}$ \\
\hline & Eurasian Tree Sparrow & Passer montanus & $\mathrm{LC}$ \\
\hline \multirow{3}{*}{$\begin{array}{l}\text { PASSERIFORMES/ } \\
\text { Motacillidae }\end{array}$} & Paddyfield Pipit & Anthus rufulus & $\mathrm{LC}$ \\
\hline & Grey Wagtail & Motacilla cinerea & LC \\
\hline & White Wagtail & Motacilla alba & $\mathrm{LC}$ \\
\hline
\end{tabular}


Table 2. Continued...

\begin{tabular}{|c|c|c|c|}
\hline ORDER/Family & Common Name & Scientific Name & $\begin{array}{l}\text { Status: } \\
\text { IUCN }\end{array}$ \\
\hline \multirow{9}{*}{$\begin{array}{l}\text { PASSERIFORMES/ } \\
\text { Fringillidae }\end{array}$} & Blyth's Rosefinch & Carpodacus grandis & $\mathrm{NE}$ \\
\hline & Pink-browed Rosefinch & Carpodacus rodochroa & $\mathrm{LC}$ \\
\hline & Vinaceous Rosefinch & Carpodacus vinaceus & $\mathrm{LC}$ \\
\hline & Common Rosefinch & Carpodacus erythrinus & $\mathrm{LC}$ \\
\hline & Brown Bullfinch & Pyrrhula nipalensis & $\mathrm{LC}$ \\
\hline & Orange Bullfinch & Pyrrhula aurantiaca & $\mathrm{LC}$ \\
\hline & Red-headed Bullfinch & Pyrrhula erythrocephala & LC \\
\hline & Plain Mountain Finch & Leucosticte nemoricola & LC \\
\hline & Yellow-breasted Greenfinch & Chloris spinoides & $\mathrm{LC}$ \\
\hline \multirow{11}{*}{$\begin{array}{l}\text { PASSERIFORMES/ } \\
\text { Emberizidae } \\
\text { PASSERIFORMES/ } \\
\text { Stenostiridae } \\
\text { PASSERIFORMES/ } \\
\text { Paridae }\end{array}$} & Crested Bunting & Melophus lathami & $\mathrm{LC}$ \\
\hline & Rock Bunting & Emberiza cia & $\mathrm{LC}$ \\
\hline & $\begin{array}{l}\text { Grey headed canary } \\
\text { flycatcher }\end{array}$ & Culicicapa ceylonensis & LC \\
\hline & Fire-capped Tit & Cephalopyrus flammiceps & $\mathrm{LC}$ \\
\hline & Coal Tit & Periparus ater & $\mathrm{LC}$ \\
\hline & Rufous-naped Tit & Periparus rufonuchalis & $\mathrm{LC}$ \\
\hline & Green-backed Tit & Parus monticolus & LC \\
\hline & Cinereous Tit & Parus cinereus & NE \\
\hline & Spot-winged Tit & Parus melanolophus & $\mathrm{NE}$ \\
\hline & Black-lored Tit & Machlolophus xanthogenys & LC \\
\hline & Yellow-cheeked Tit & Machlolophus spilonotus & LC \\
\hline \multirow{3}{*}{$\begin{array}{l}\text { PASSERIFORMES/ } \\
\text { Pnoepygidae } \\
\text { PASSERIFORMES/ } \\
\text { Hirundinidae }\end{array}$} & $\begin{array}{l}\text { Scaly breasted Wren } \\
\text { Babbler }\end{array}$ & Pnoepyga albiventer & LC \\
\hline & Nepal House Martin & Delichon nipalense & $\mathrm{LC}$ \\
\hline & Barn Swallow & Hirundo rustica & $\mathrm{LC}$ \\
\hline \multirow{3}{*}{$\begin{array}{l}\text { PASSERIFORMES/ } \\
\text { Pycnonotidae }\end{array}$} & Black Bulbul & Hypsipetes leucocephalus & $\mathrm{LC}$ \\
\hline & Himalayan Bulbul & Pycnonotus leucogenis & $\mathrm{LC}$ \\
\hline & Red-vented Bulbul & Pycnonotus cafer & $\mathrm{LC}$ \\
\hline \multirow{9}{*}{$\begin{array}{l}\text { PASSERIFORMES/ } \\
\text { Phylloscopidae }\end{array}$} & Buff-barred Warbler & Phylloscopus pulcher & $\mathrm{LC}$ \\
\hline & Ashy-throated Warbler & Phylloscopus maculipennis & $\mathrm{LC}$ \\
\hline & Plain Leaf Warbler & Phylloscopus neglectus & LC \\
\hline & Hume's Warbler & Phylloscopus humei & $\mathrm{LC}$ \\
\hline & Tickell's Leaf Warbler & Phylloscopus affinis & $\mathrm{LC}$ \\
\hline & Greenish Leaf Warbler & Phylloscopus trochiloides & $\mathrm{LC}$ \\
\hline & Large-billed Leaf Warbler & Phylloscopus magnirostris & $\mathrm{LC}$ \\
\hline & Yellow-vented Leaf Warbler & Phylloscopus cantator & $\mathrm{LC}$ \\
\hline & Grey-hooded Leaf Warbler & Phylloscopus/xanthoschistos & LC \\
\hline \multirow{6}{*}{$\begin{array}{l}\text { PASSERIFORMES/ } \\
\text { Scotocercidae } \\
\text { PASSERIFORMES/ } \\
\text { Aegithalidae } \\
\text { PASSERIFORMES/ } \\
\text { Zosteropidae }\end{array}$} & Black-faced Warbler & Abroscopus schisticeps & $\mathrm{LC}$ \\
\hline & Aberrant Bush Warbler & Horornis flavolivaceus & LC \\
\hline & Black-throated Tit & Aegithalos concinnus & $\mathrm{LC}$ \\
\hline & White-throated Tit & Aegithalos niveogularis & $\mathrm{LC}$ \\
\hline & Whiskered Yuhina & Yuhina flavicollis & $\mathrm{LC}$ \\
\hline & Oriental White-eye & Zosterops palpebrosus & $\mathrm{LC}$ \\
\hline
\end{tabular}


Table 2. Continued...

\begin{tabular}{|c|c|c|c|}
\hline ORDER/Family & Common Name & Scientific Name & $\begin{array}{l}\text { Status: } \\
\text { IUCN }\end{array}$ \\
\hline \multirow{6}{*}{$\begin{array}{l}\text { PASSERIFORMES/ } \\
\text { Leiothrichidae }\end{array}$} & Striated Laughing thrush & Grammatoptila striata & LC \\
\hline & $\begin{array}{l}\text { White-throated Laughing- } \\
\text { thrush }\end{array}$ & Garrulax albogularis & $\mathrm{LC}$ \\
\hline & Streaked Laughing-thrush & Trochalopteron lineatum & $\mathrm{LC}$ \\
\hline & $\begin{array}{l}\text { Chestnut-crowned Laugh- } \\
\text { ing-thrush }\end{array}$ & Trochalopteron erythrocephalum & $\mathrm{LC}$ \\
\hline & Rufous Sibia & Heterophasia capistrata & $\mathrm{LC}$ \\
\hline & Chestnut-tailed Minla & Chrysominla strigula & LC \\
\hline $\begin{array}{l}\text { PASSERIFORMES/ } \\
\text { Regulidae }\end{array}$ & Goldcrest & Regulus regulus & LC \\
\hline \multirow{3}{*}{$\begin{array}{l}\text { PASSERIFORMES/ } \\
\text { Certhiidae }\end{array}$} & Bar-tailed Treecreeper & Certhia himalayana & LC \\
\hline & Eurasian Treecreeper & Certhia familiaris & $\mathrm{LC}$ \\
\hline & Hodgson's Treecreeper & Certhia hodgsoni & $\mathrm{LC}$ \\
\hline $\begin{array}{l}\text { PASSERIFORMES/ } \\
\text { Sittidae }\end{array}$ & White-tailed Nuthatch & Sitta himalayensis & $\mathrm{LC}$ \\
\hline \multirow{3}{*}{$\begin{array}{l}\text { PASSERIFORMES/ } \\
\text { Sturnidae }\end{array}$} & Common Myna & Acridotheres tristis & $\mathrm{LC}$ \\
\hline & Jungle Myna & Acridotheres fuscus & $\mathrm{LC}$ \\
\hline & Hill Myna & Gracula religiosa & $\mathrm{LC}$ \\
\hline \multirow{21}{*}{$\begin{array}{l}\text { PASSERIFORMES/ } \\
\text { Muscicapidae }\end{array}$} & Oriental Magpie Robin & Copsychus saularis & $\mathrm{LC}$ \\
\hline & Dark-sided Flycatcher & Muscicapa sibirica & $\mathrm{LC}$ \\
\hline & Rusty-tailed Flycatcher & Muscicapa ruficauda & $\mathrm{LC}$ \\
\hline & Rufous-bellied Niltava & Niltava sundara & $\mathrm{LC}$ \\
\hline & Asian Verditer Flycatcher & Eumyias thalassinus & $\mathrm{LC}$ \\
\hline & Indian Blue Robin & Larvivora brunnea & $\mathrm{LC}$ \\
\hline & Little Forktail & Enicurus scouleri & $\mathrm{LC}$ \\
\hline & Spotted Forktail & Enicurus maculatus & $\mathrm{LC}$ \\
\hline & Blue-fronted Robin & Cinclidium frontale & $\mathrm{LC}$ \\
\hline & Blue Wshistling Thrush & Myophonus caeruleus & $\mathrm{LC}$ \\
\hline & Himalayan Bluetail & Tarsiger rufilatus & $\mathrm{LC}$ \\
\hline & Ultramarine Flycatcher & Ficedula superciliaris & $\mathrm{LC}$ \\
\hline & Little Psied Flycatcher & Ficedula westermanni & $\mathrm{LC}$ \\
\hline & $\begin{array}{l}\text { White-capped Water Red- } \\
\text { start }\end{array}$ & Chaimarrornis leucocephalus & $\mathrm{LC}$ \\
\hline & Blue-fronted Redstart & Phoenicurus frontalis & $\mathrm{LC}$ \\
\hline & Blue-capped Redstart & Phoenicurus coeruleocephala & $\mathrm{LC}$ \\
\hline & Black Redstart & Phoenicurus ochruros & LC \\
\hline & $\begin{array}{l}\text { Chestnut-bellied Rock } \\
\text { Thrush }\end{array}$ & Monticola rufiventris & $\mathrm{LC}$ \\
\hline & Blue Rock Thrush & Monticola solitarius & $\mathrm{LC}$ \\
\hline & Pied Bush Chat & Saxicola caprata & $\mathrm{LC}$ \\
\hline & Desert Wheatear & Oenanthe deserti & $\mathrm{LC}$ \\
\hline
\end{tabular}


Table 2. Continued...

\begin{tabular}{|c|c|c|c|}
\hline ORDER/Family & Common Name & Scientific Name & $\begin{array}{l}\text { Status: } \\
\text { IUCN }\end{array}$ \\
\hline \multirow{10}{*}{$\begin{array}{l}\text { PASSERIFORMES/ } \\
\text { Turdidae }\end{array}$} & Long-tailed Thrush & Zoothera dixoni & $\mathrm{LC}$ \\
\hline & Plain-backed Thrush & Zoothera mollissima & $\mathrm{LC}$ \\
\hline & Long-billed Thrush & Zoothera monticola & LC \\
\hline & Scaly Thrush & Zoothera dauma & $\mathrm{LC}$ \\
\hline & Mistle Thrush & Turdus viscivorus & $\mathrm{LC}$ \\
\hline & Grey-winged Blackbird & Turdus boulboul & $\mathrm{LC}$ \\
\hline & Tickell's Thrush & Turdus unicolor & $\mathrm{LC}$ \\
\hline & White-collared Blackbird & Turdus albocinctus & $\mathrm{LC}$ \\
\hline & Chestnut Thrush & Turdus rubrocanus & $\mathrm{LC}$ \\
\hline & Red-throated Thrush & Turdus ruficollis & $\mathrm{LC}$ \\
\hline \multirow{10}{*}{$\begin{array}{l}\text { PASSERIFORMES/ } \\
\text { Troglodytidae } \\
\text { PICIFORMES/ Ram- } \\
\text { phastidae } \\
\text { PICIFORMES/ Pici- } \\
\text { dae }\end{array}$} & Winter Wren & Troglodytes troglodytes & $\mathrm{LC}$ \\
\hline & Great Barbet & Psilopogon virens & $\mathrm{LC}$ \\
\hline & Black rumped Woodpecker & Dinopium benghalense & LC \\
\hline & $\begin{array}{l}\text { Greater Yellow naped } \\
\text { Woodpecker }\end{array}$ & Chrysophlegma flavinucha & $\mathrm{LC}$ \\
\hline & $\begin{array}{l}\text { Lesser Yellow naped Wood- } \\
\text { pecker }\end{array}$ & Picus chlorolophus & $\mathrm{LC}$ \\
\hline & Grey-headed Woodpecker & Picus canus & $\mathrm{LC}$ \\
\hline & Scaly-bellied Woodpecker & Picus squamatus & $\mathrm{LC}$ \\
\hline & Brown-fronted Woodpecker & Dendrocopos auriceps & $\mathrm{LC}$ \\
\hline & Himalayan Woodpecker & Dendrocopos himalayensis & $\mathrm{LC}$ \\
\hline & Rufous-bellied Woodpecker & Dendrocopos hyperythrus & $\mathrm{LC}$ \\
\hline \multirow{4}{*}{$\begin{array}{l}\text { PSITTACIFORMES/ } \\
\text { Psittaculidae }\end{array}$} & Slaty-headed Parakeet & Psittacula himalayana & $\mathrm{LC}$ \\
\hline & Blossom-headed Parakeet & Psittacula roseata & NT \\
\hline & Red-Breasted Parakeet & Psittacula alexandri & NT \\
\hline & Plum-headed Parakeet & Psittacula cyanocephala & LC \\
\hline \multirow{3}{*}{$\begin{array}{l}\text { STRIGIFORMES/ } \\
\text { Strigidae }\end{array}$} & Collared Owlet & Glaucidium brodiei & LC \\
\hline & Asian Barred Owlet & Glaucidium cuculoides & $\mathrm{NE}$ \\
\hline & Brown Wood Owl & Strix leptogrammica & $\mathrm{LC}$ \\
\hline
\end{tabular}

(Abbreviations used: Status as per the IUCN Red List of Threatened Species, NT $=$ Near Threatened, E = Endangered, CE $=$ Critically Endangered, VU= Vulnerable, $\mathrm{LC}=$ Least Concerened and NE = Not Evaluated)

The charismatic wildlife species inhabiting Binsar is the leopard (Panthera pardus) and is the top predator here (Bhalla et al., 2015). Kala and Kothari (2013) reported nine species of mammals and birds each from the BWLS. Rawat et al. (2013) reported four species of mammals and two species of birds. Khan et al. (2000), Majila and Kala (2010) and Bhalla et al. (2015) reported 10 species of birds and mammals from the BWLS. BWLS is rich in avian diversity, it has been declared as an important bird area by BirdLife International with over 160 species. BWLS is an important Bird Area under the A3 Category of Biome 08 (BirdLife International, 2021). The sanctuary also harbors 166 species of birds 
(Uttarakhand Forest Department, 2014). Mohan and Sondhi (2014, 2015, 2017) published three updated checklists of the birds of Uttarakhand listing 686, 693 and 710 species of birds. Bhalla et al. (2020) documented some previously recorded birds and mammal species and added a single species of Indian porcupine (Hystrix indica) to BWLS. Sultana and Khan (2000) studied the birds of oak forest in the Kumaun Himalaya and documented a total of 382 bird species from Kumaun Himalaya including species from Almora (182 birds), Nainital (81 birds), Pithoragarh (162 birds) districts respectively. Shahabuddin et al. (2017) documented 136 species of birds from banjoak- chirpine forest of Nainital and Almora Districts of Kumaon, Uttarakhand. In a more recent years, Lepage (2021) documented checklist of 165 species of birds belonging to 12 orders and 44 families in the Avibase, the word bird database of Binsar Wildlife Sanctuary, Almora.

\section{Insect diversity}

The sanctuary also harbors major group of insects including butterflies, moths, beetles, bees, grasshoppers, dragon flies, bugs and dipteran flies. A total of 121 species of insects belonging to 36 families and eight orders were reported from BWLS. Total number of species of different insect orders and their percent contribution to total number of species recorded from BWLS is presented in Figure 3. Order Lepidoptera was the most dominant order with 50 species and amounting to $41.32 \%$ of the total number of species of insects, followed by Coleoptera (25) amounting to $20.66 \%$, Hymenoptera (14) amounting to $11.57 \%$, Orthoptera (10) amounting to $8.26 \%$, Odonata (8) amounting to $6.61 \%$, Diptera (7) amounting to $5.79 \%$, Hemiptera (6) amounting to $4.96 \%$ and Dictyoptera with a single species.

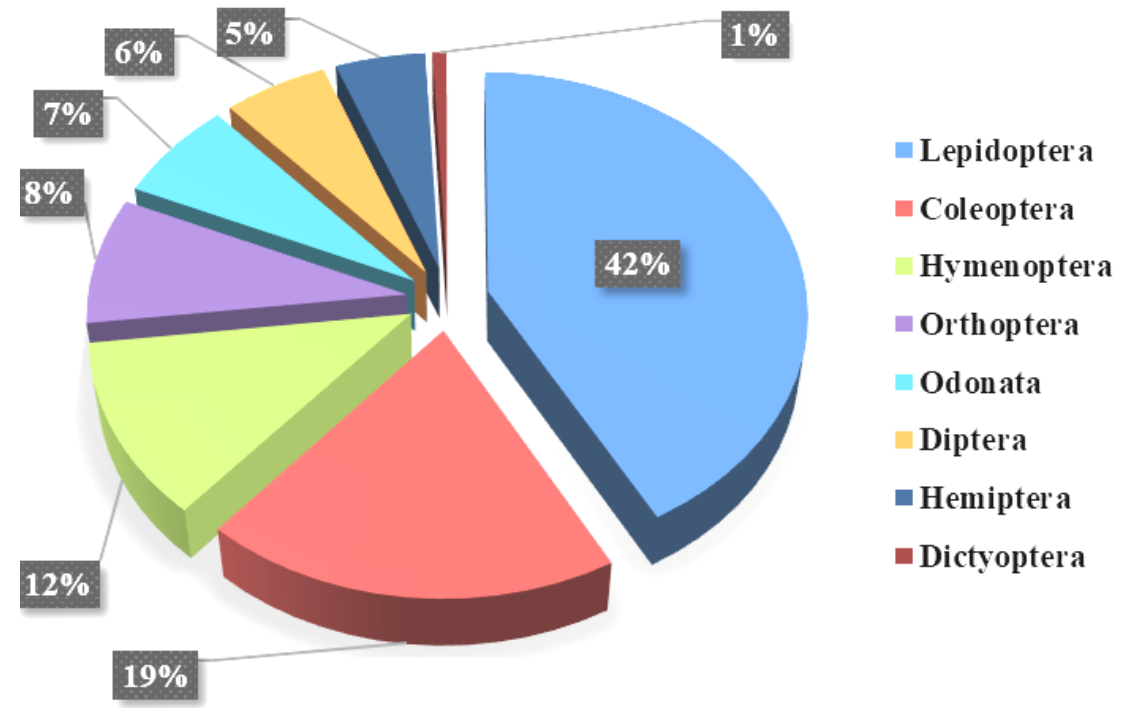

Figure 3. Total number of species of different insect orders and their percent contribution to total number of species recorded from BWLS. 
Order Lepidoptera: Lepidoptera was the most dominant insect order in the sanctuary, represented by 50 species under eight families (Table 3). Five species namely Neptis sankara (under Schedule I), Neptis zaida, Callerebia scanda (under Schedule II), Euploea core, Aporia agathon (under Schedule IV) are legally protected under the Indian Wildlife (Protection) Act, 1972. On the basis of total number of species, Nymphalidae was the most dominant family of this order with 23 species followed by Pieridae (12), Lycaenidae (4), Papilionidae, Riodinidae (three each), Hesperiidae (2), Erebidae, Noctuidae and Sphingidae (one each), respectively. Status of species of Lepidopteran insects was assigned as Very Common (VC) when counted in large numbers of individuals, Common (C) when observed regularly, Uncommon (UC) when recorded occasionally and Rare (R) when recorded rarely (Table 3 and Figure 4).

Order Coleoptera: Coleoptera was the second most dominant insect order in the BWLS and represented 25 species under six families. Species composition of coleopteran insects of the BWLS has been shown in the Table 4. On the basis of the total number of species, Scarabaeidae was the most dominant family of this order with 12 species followed by Chrysomelidae (5), Coccinellidae, Dytiscidae, Meloidae (2 species each), Lagriidae and Tenebrionidae (one each), respectively.

Other minor insect orders: Table 5 shows the species composition of other minor group of insect orders reported from the BWLS. Hymenoptera was the third most dominant insect order in the sanctuary and belonging to 14 species under seven families. On the basis of the total number of species, Apidae was the most dominant family of this order with six species followed by Ichneumonidae, Scoliidae (two each), Pompilidae, Sphecidae, Vespidae and Xylocopidae (one each), respectively. Orthoptera was represented by only two families. Acrididae was the dominant family with eight species, while Tettigonidae was represented by two species.

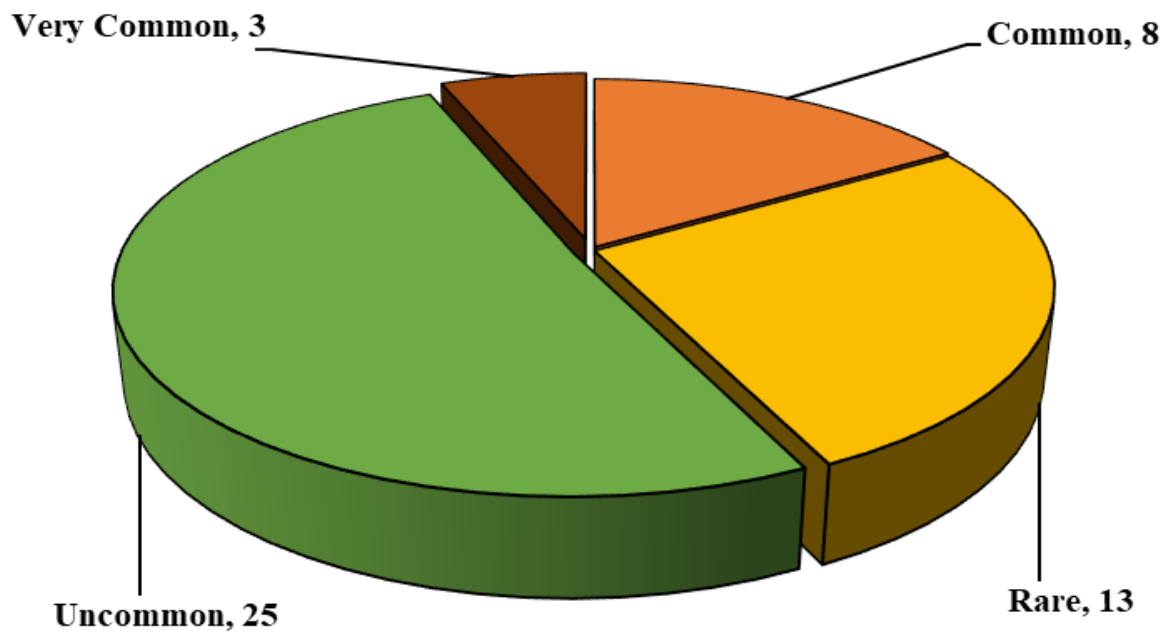

Figure 4. Status of species of Lepidopteran insects recorded from BWLS 
Table 3. Species composition of Lepidopteran fauna of Binsar Wildlife Sanctuary.

\begin{tabular}{|c|c|c|c|}
\hline Family & Common Name & Species Name & $\begin{array}{l}\text { Local } \\
\text { status }\end{array}$ \\
\hline \multirow[t]{23}{*}{ Nymphalidae } & Yellow Coster & Acraea issoria anamala Kollar & $\mathrm{R}$ \\
\hline & Indian Tortoiseshell & Aglais cashmirensis (Fruhstorfer) & $\mathrm{VC}$ \\
\hline & Large Silverstripe & Argynnis childreni (Grey) & $\mathrm{UC}$ \\
\hline & Indian Fritillary & Argynnis hyperbius Linnaeus & $\mathrm{C}$ \\
\hline & Common Satyr & Aulocera swaha Kollar & $\mathrm{R}$ \\
\hline & Great Satyr & Aulocera padma Kollar & $\mathrm{R}$ \\
\hline & Ringed Argus & Callerebia annada (Moore) & $\mathrm{R}$ \\
\hline & Pallid Argus & Callerebia scanda (Kollar)* & $\mathrm{UC}$ \\
\hline & Plain Tiger & Danaus chrysippus (Linnaeus) & UC \\
\hline & Common Crow & Euploea core (Cramer)* & $\mathrm{UC}$ \\
\hline & Chocolate Pansy & Junonia iphita Cramer & $\mathrm{C}$ \\
\hline & Orange Oakleaf & Kallima inachus Boisduval & $\mathrm{UC}$ \\
\hline & Blue Admiral & Kaniska canace (Linnaeus) & $\mathrm{R}$ \\
\hline & Common Wall & Lasiommata schakra (Kollar) & $\mathrm{R}$ \\
\hline & Straight-Banded Treebrown & Lethe verma Kollar & UC \\
\hline & Broad-Banded Sailer & Neptis sankara (Kollar)* & $\mathrm{UC}$ \\
\hline & Pale Green Sailer & Neptis zaida Westwood* & $\mathrm{R}$ \\
\hline & Tabby & Pseudergolis wedah (Kollar) & $\mathrm{UC}$ \\
\hline & Common Leopard & Phalanta phalantha (Drury) & $\mathrm{UC}$ \\
\hline & Western Courtier & Sephisa dichroa (Kollar) & $\mathrm{UC}$ \\
\hline & Painted Lady & Vanessa cardui Linnaeus & UC \\
\hline & Indian Red Admiral & Vanessa indica Herbst & VC \\
\hline & Large Three-Ring & Ypthima nareda nareda (Kollar) & UC \\
\hline \multirow[t]{12}{*}{ Pieridae } & Pioneer & Belenois aurota (Fabricius) & $\mathrm{UC}$ \\
\hline & Common Emigrant & Catopsilia pomona Linnaeus & $\mathrm{C}$ \\
\hline & Dark Clouded Yellow & Colias fieldii Menetries & $\mathrm{C}$ \\
\hline & Small Grass Yellow & Eurema brigitta rubella Wallace & $\mathrm{UC}$ \\
\hline & Common Grass Yellow & Eurema hecabe Linnaeus & C \\
\hline & Spotless Grass Yellow & Eurema laeta Boisduval & UC \\
\hline & Himalayan Brimstone & Gonepteryx rhamni nepalensis Linnaeus & $\mathrm{C}$ \\
\hline & Great Blackvein & Aporia agathon agathon* (Gray) & UC \\
\hline & Great Blackvein & Aporia agathon phryxe (Boisduval) & $\mathrm{R}$ \\
\hline & Large Cabbage White & Pieris brassicae Linnaeus & $\mathrm{C}$ \\
\hline & Indian Cabbage White & Pieris canidia indica Evans & VC \\
\hline & Bath White & Pontia daplidice (Linnaeus) & $\mathrm{R}$ \\
\hline \multirow[t]{4}{*}{ Lycaenidae } & Sorrel Sapphire & Heliophorus sena Kollar & $\mathrm{UC}$ \\
\hline & White-Bordered Copper & Lycaena panava (Kollar) & UC \\
\hline & Red Pierrot & Talicada nyseus (Guerin-Meneville) & $\mathrm{UC}$ \\
\hline & Pale Hedge Blue & Udara dilectus Moore & $\mathrm{R}$ \\
\hline
\end{tabular}


Table 3. Continued...

\begin{tabular}{llll}
\hline Family & Common Name & Species Name & $\begin{array}{l}\text { Local } \\
\text { status }\end{array}$ \\
\hline Papilionidae & Common Windmill & Byasa polyeuctes letincius (Fruhstorfer) & $\mathrm{UC}$ \\
& Common Peacock & Papilio bianor polyctor Boisduval & $\mathrm{UC}$ \\
\multirow{5}{*}{ Riodindae } & Common Mormon & Papilio polytes Linnaeus & $\mathrm{UC}$ \\
& Common Punch & Dodona durga durga (Kollar) & $\mathrm{UC}$ \\
& Tailed Punch & Dodona eugenes Bates & $\mathrm{R}$ \\
Hesperiidae & Mixed Punch & Dodona ouida Hewitson & $\mathrm{UC}$ \\
& Himalayan Darter & Ochlodes brahma Moore & $\mathrm{R}$ \\
Erebidae & Evan's Snow Flat & Tagiades cohaerens Cynthia Evans & $\mathrm{R}$ \\
& - & Calpe ophideroides Guenee & $\mathrm{R}$ \\
Sphingidae & Handmaiden moth & Syntomoides imaon Cramer & $\mathrm{C}$ \\
\hline
\end{tabular}

(Abbreviations used: VC = Very Common, $\mathrm{C}=\mathrm{Common}, \mathrm{UC}=$ Uncommon, $\mathrm{R}=$ Rare and * indicates legally protected species under the Indian Wildlife (Protection) Act, 1972)

Odonata was represented by eight species under four families. On the basis of the total number of species, Libellullidae was the most dominant family of this order with five species followed by Aeschnidae, Euphaeidae and Synlestidae (one each), respectively. Diptera was represented by seven species under four families. Tabanidae was the most dominant family of this order with three species followed by Asilidae (2), Syrphidae and Tipulidae (one each), respectively. Hemiptera was represented by six species under three families. On the basis of the total number of species, Pentatomidae was the most dominant family of this order with three species followed by Coreidae (2) and Lygaeidae (1). Order Dictyoptera was represented by single family Mantidae with species Deiphobe infuscate Saussure. Figure 5 shows some images of insects recorded in BWLS.
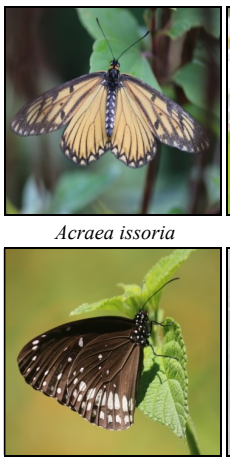

Euploea core

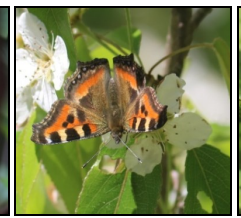

Aglais cashmirensis

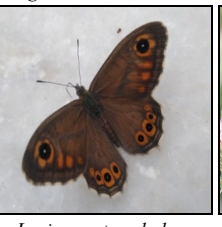

Lasiommata schakra

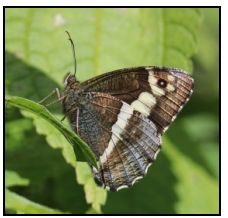

Aulocera swaha

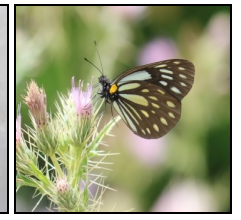

Aporia agathon

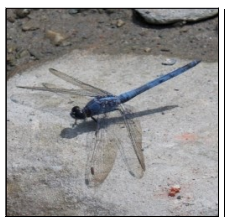

Orthetrum taeniolatum

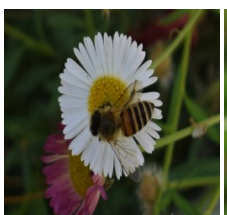

Apis cerana

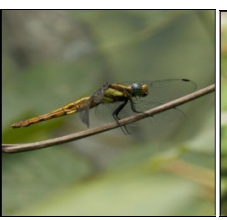

Orthetrum glaucum

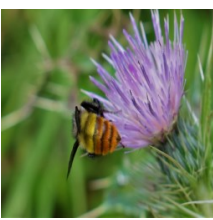

Bombus sp.

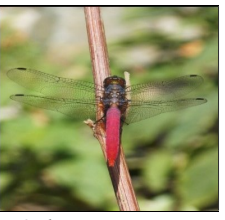

Orthetrum pruinosum

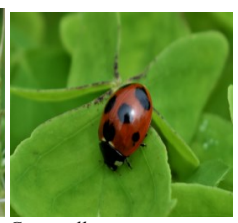

Figure 5. Examples of insect fauna reported from BWLS 
Table 4. Species composition of coleopteran insects of Binsar Wildlife Sanctuary.

\section{ORDER: COLEOPTERA}

Family: Scarabaeidae

Anomala lineatopennis Blanchard

Anomala sp.

Gymnopleurus subtilis Walker

Jumnos roylei Hope

Lachnosterna cavifrons Brenske

Lytta limbata Redtenbacher

Onthophagus gagates Hope

O. rubricollis Hope

Protaetia neglacta Hope

P. pretiosa Nonfried

Pseudolucanus cantor Hope

Scarites sulcatus Olivier

Family: Chrysomelidae

Altica himensis Shukla

Gallerucida rutilans Hope
Meristata sexmaculata (Kollar \& Redtenbacher)

Merista tatrifasciata Hope

Mimastra sp.

Family: Coccinellidae

Coccinella septumpunctata Linnaeus

Haluzia sanscrieta Muls.

Family: Meloidae

Mylabris cichorii Linnaeus

Mylabris sp.

Family: Lagriidae

Cerogria nepalensis Hope

Family: Tenebrionidae

Cistelomorpha sp.

Family: Dytiscidae

Agabus amoenus sinuaticollis Regimbart Agabus biguttatus (Oliver)

Table 5. Species composition of minor groups of insect orders of Binsar Wildlife Sanctuary.

\begin{tabular}{ll}
\hline ORDER: HYMENOPTERA & ORDER: ODONATA \\
\hline Family: Apidae & Family: Libellullidae \\
Anthophora confuse Smith & Crocothemis servilia servilia (Drury) \\
Apis cerana Fabricius & Orthetrum sabina sabina (Drury) \\
Apis laboriosa Smith & O. glaucum Brauer \\
Bombus sp. & O. pruinosum neglectum (Rambur) \\
Bremus sp. & O. taeniolatum (Schneider) \\
Crocisa ramose Lepeletier & Family: Aeschnidae \\
Family: Ichneumonidae & Anax immaculiforns Rambur \\
Ichneumon sp. & Family: Euphaeidae \\
Ophion sp. & Bayadera indica (Selys) \\
Family: Scoliidae & Family: Synlestidae \\
Compsomeris asiatica himalaya Bar. & Megalestes major Selys \\
Scolia venusta Smith & ORDER: DIPTERA \\
Family: Pompilidae & Family: Tabanidae \\
Salius flavus Fabricius & Pangonia longirostris Hardwicke \\
Family: Sphecidae & Philoliche sp. \\
Ammophila punctata Smith & Tabanus orientis Walker \\
Family: Vespidae & Family: Asilidae \\
Vespa sp. & Philodious javanus Wied. \\
Family: Xylocopidae & Stenopogano ldroydi Josephs \& Pauri \\
Xylocopa fenestrate Fabricius & Family: Syrphidae \\
\hline
\end{tabular}


Table 5. Continued...

\begin{tabular}{ll}
\hline ORDER: ORTHOPTERA & Syrphus fulvifacies Brunetti \\
\hline Family: Acrididae & Family: Tipulidae \\
Chorthippus almoranus Uvarov & Tipula himalayensis Brunetti \\
Gastrimargus transversus Thunberg & ORDER: HEMIPTERA \\
Heteropternis respondence (Walker) & Family: Pentatomidae \\
Paraconophyma scabra Walker & Erthesina fullo Thunberg \\
Patanga japonica (Bolivar) & Dalpada sp. \\
Pternoscirta cinctifemur Walker & Nezara viridula Linnaeus \\
Spathosternum p. prasiniferum (Walker) & Family: Coreidae \\
Xenocatantops karnyi Kirby & Cletus punctulatus Westwood \\
Family: Tettigonidae & Ochrochira albiditarsis Westwood \\
Himertula kinneri Uvarov & Family: Lygaeidae \\
Letana linearis (Walker) & Lygaeus equestris Linnaeus \\
\hline
\end{tabular}

BWLS harbors major two groups of insects i.e. butterflies and beetles and minor group of insects including bees, bumble-bees, carpenter bees, dragon flies, bugs and dipteran flies. In comparison, Ilyas (1998) documented two species of butterflies, Mixed Punch (Dodona ouida Hewitson) and Great Satyr (Aulocera padma Kollar) from the BWLS. In the recent years, Ghosh et al. (2011) and Ghosh et al. (2018) reported two species of aquatic beetles belonging to family Dytiscidae from the BWLS. Arya et al. (2016a) studied the distribution and diversity of beetles (Insecta: Coleoptera) in different elevational zones of BWLS, Almora, Uttarkhand, India and reported 23 species of beetles from 18 genera and six families. Arya et al. (2017) studied the population ecology and bioenergetics of Chorthippus almoranus Uvarov (Orthoptera: Acrididae) of the BWLS. Tamta (2017) recorded 115 species of insects belonging to eight orders from different locations of BWLS. Arya et al. (2018a) reported 53 species of anthophilous insects belonging to 18 families under four orders facilitating the pollination process in the entire area of the BWLS. Arya et al. (2018b) studied the bio-spectrum of different groups of insect orders and reported a total of 115 species of insects belonging to eight orders was sorted into four categories based on their major feeding habits viz., herbivorous, omnivorous, predators and saprophagous in the BWLS, Western Himalayas. In a more recent study, Arya et al. (2020) studied the diversity of butterflies (Lepidoptera: Papilionoidea) in a temperate forest ecosystem in the BWLS and reported 46 species and 35 genera under six families 


\section{Conclusion}

BWLS is a low profiled protected area in terms of adopted biodiversity conservation and management strategies, and it is still unexplored or under-explored in relation to faunal diversity. The provided comprehensive checklist on faunal diversity chiefly mammals, Aves and different groups of insects is intended to serve as a reliable biodiversity data source for biological studies and will be helpful in biodiversity conservation and management plans as well as for monitoring faunistic changes which might occur as we move further deeper towards the Anthropocene in the $21^{\text {st }}$ century.

\section{Acknowledgement}

Authors would like to thank scientists, entomologists, naturalists, and other researchers who had contributed much to our understandings on floral and faunal diversity in Binsar Wildlife Sanctuary. Their potential efforts made us possible to provide comparative knowledge on faunal species richness in the sanctuary. Sincere thanks to anonymous reviewers for their critical comments that helped in improving the manuscript.

\section{References}

Arya, M.K. and Verma, A. (2020). An insight into the Butterflies (Lepidoptera, Papilionoidea) associated with protected area network of Uttarakhand, Western Himalaya, India. In: Rathoure, A.K. and Chauhan, P. B. Current State and Future Impacts of Climate Change on Biodiversity. AEEGT Book Series, IGI Global, United States of America. pp. 154-178.

Arya, M.K., Tamta, P. and Dayakrishna (2016a). Study on distribution and diversity of beetles (Insecta: Coleoptera) in different elevational zones of Binsar Wildlife Sanctuary, Almora, Uttarkhand, India. Journal of Entomology and Zoology Studies, 4(4): 311-316.

Arya, M.K., Tamta, P. and Dayakrishna (2016b). Species composition, distribution and diversity of butterflies along altitudinal gradients in Binsar Wildlife Sanctuary, Kumaun Himalayas, Uttarakhand, India. In: M. K. Arya, P. Kumar and R. Joshi (Eds). Biological diversity \& Ecology. Discovery Publishing House Pvt. Ltd. New Delhi (India). pp. 1-11.

Arya, M.K., Tamta, P. And Verma, A. (2018a). Systematic survey on alpha diversity of anthophilous insect fauna in Binsar Wildlife Sanctuary, Western Himalaya. Entomon, 43(2): 99-110.

Arya, M.K., Tamta, P., Dayakrishna and Joshi, P.C. (2017). Observation on Population ecology and Bioenergetics of Chorthippus almoranus Uvarov (Orthoptera: Acrididae). Journal of Environment and Bio-Sciences, 31(1): 101-107.

Arya, M.K., Tamta, P., Kumar, K. and Joshi, P.C. (2018b). Bio-spectrum of trophic position and secondary productivity of insects of Binsar Wildlife Santuary in the Western Himalayas. Journal of Environment and Bio- Sciences, 32(1): 35-43.

Arya, M.K., Verma, A. and Badoni, A. (2019). A checklist on beetles diversity (Coleoptera: Insecta) associated with the protected areas of Uttarakhand, Western Himalaya. In: Rashmi Tripathi, Vijay Sharma and P. Kumar (eds). Animal Biodiversity and Fisheries. Discovery Publishing House Pvt. Ltd. New Delhi (India). pp. 1-26

Arya, M.K., Verma, A. and Tamta, P. (2020). Diversity of butterflies (Lepidoptera: Papilionoidea) in a temperate forest ecosystem, Binsar Wildlife Sanctuary, Indian Himalayan Region. Nature Environment and Pollution Technology, 19(3): 1133-1140.

Bhalla, P., Bhattacharya, P. and Gupta, N.C. (2015). Sound levels assessment in an ecotourism destination: A case study on Binsar Wildlife Sanctuary of Indian Himalayan Region. International Journal of Scientific and Research Publications, 5(7): 7.

Bhalla, P., Bhattacharya, P., Areendran, G. and Raj, K. (2020). Ecotourism spatio- temporal Models to identify visitation pat- 
terns across the Indian Himalayan Region. Geo Journal, https://doi.org/10.1007/s10708-020-10336-8

Bhalla, P., Coghlam, A. and Bhattacharya, P. (2017). Case Study-Development of community-based ecotourism in Binsar Wildlife Sanctuary of Indian Himalayan Region. World Tourism Organization and Griffith University, Managing Grwoth and Sustainable Tourism Governance in Asia and the Pacificn, Madrid: UNWTO Publications. pp. 54-60.

Binsar Management Plan (2000-2010). Management Plan of Binsar Wildlife Sanctuary, Wildlife Preservation Organization, Uttar Pradesh.

BirdLife International (2021). Important bird and biodiversity area factsheet: Binsar Wildlife Sanctuary. https:// www.birdlife.org. [Accessed on 25/05/2021].

Gansser, A. (1964). Geology of the Himalayas. London: John Wiley and Sons.

Ghosh, S. K., Chakraborti, U., Pati, P. and Mitra, B. (2018). Similarity analysis in species composition of the aquatic beetle fauna among some Indian Protected Areas in respect to Satkosia and Baisipalli Wildlife Sanctuary, Odisha. Ambient Science, 5(2): 25-30.

Ghosh, S. K., Ghosh, P. and Mitra, B. (2011). A preliminary report on the predaceous diving beetles (Dytiscidae: Coleoptera) of Binsar Wildlife Sanctuary, Uttarakhand. Bug 'R' All Newsletter, 17: 19-20.

Grimmett, R., Inskipp, C. and Inskipp, T. (2011). Birds of the Indian Subcontinent. Oxford University Press, UK.

Ilyas, O. (1998). People and protected areas: The case of Binsar Wildlife Sanctuary. Published by World Wide Fund for Nature India, Lodhi state, Max Mullar Marg, New Delhi-110003.

Ilyas, O. (1999). Sighting of Cheer Pheasant (Catreus wallichii) at Binsar Wildlife Sanctuary, Almora, U.P. World Pheasant Association-SARO News, 1(1): 7.

Ilyas, O. and Khan, J.A. (2001). Birds of Binsar. Newsletter for Birdwatcher, 41(1): 61-64.

Ilyas, O. and Khan, J.A. (2004). Food habits of barking deer (Muntiacus muntjak) and Goral (Naemorheus goral) in Binsar Wildlife Sanctuary, India. Mammalia, 68(1): 1-11.

Ilyas, O. And Khan, J.A. (2005). Assessment of tree mortality and post fire vegetation pattern in Binsar Wildlife Sanctuary, Kumaon Himalaya. Tropical Ecology, 46(2): 157-163.

Islam, Z. and Rahmani, A.R. (2004). Important Bird Areas in India. Priority sites for conservation. I-xvii, 1, 1133. Indian Bird Conservation Network: Bombay Natural History Society and BirdLife International (UK), Mumbai.

IUCN (2007). IUCN Red list of Threatened species. http://www.iucnredlistorg/

Kala, C. P. and Majila, B. S. (2013). Status of forest and wildlife in the Binsar Wildlife Sanctuary, Uttarakhand, India. In C. P. Kala and C. S. Silori (Eds), Biodiversity, communities and climate change, New Delhi: Teri Publications. pp. 20-25.

Kala, C.P. and Kothari, K.K. (2013). Livestock predation by common Leopard in Binsar Wildlife Sanctuary, India: Humanwildlife conflicts and conservation issues. Human-Wild interactions, 7(2): 325-333.

Khan, A.H. and Arya, D. (2017). Analysis of forest vegetation in Binsar Wildlife Sanctuary, Kumaun Himalaya, Uttarakhand, India. American-Eurasian Journal of Agricultural \& Environmental Sciences, 17(4): 336-342.

Khan, J.A., Sultana, A. and Hussain, M.S. (2000). A study of threats to biodiversity conservation of middle altitude oak forest in Kumaon Himalaya. Final Technical Report. Department of Wildlife Sciences, Aligarh Muslim University, Aligarh, India.

Lepage, D. (2021). Checklist of the birds of Binsar Wildlife Sanctuary, Almora. Avibase, the world bird database. Retrieved from. https://avibase.bsceoc.org/checklist.jsp?lang=EN\&region=inwhuk10\&list=howardmoore\&format=1[ access on 25/02/2021].

Majila, B.S. (1992). Phytosociology, Biomass structure and primary productivity of oak-pine forest of Kumaun Himalayas. Ph.D. Thesis, Kumaun University, Nainital.

Majila, B.S. and Kala, C.P. (2010). Forest Structure and Regeneration along the altitudinal gradient in the Binsar Wildlife Sanctuary, Uttarakhand Himalaya in India. Russian Journal of Ecology, 41(1): 75-83.

Majila, B.S., Joshi, G.C. and Kala, C.P. (2005). Patterns of litter fall and litter decomposition along an altitudinal gradient in the Binsar Wildlife Sanctuary, Central Himalayas. International Journal of Sustainable Development and World Ecology, 12(2): 205-212.

Mohan, D. and Sondhi, S. (2014). An Updated Checklist of the Birds of Uttarakhand. Published by Uttarakhand Forest De- 
partment, Dehradun.

Mohan, D. and Sondhi, S. (2015). An Updated Checklist of the Birds of Uttarakhand. Published by Uttarakhand Forest Department, Dehradun.

Mohan, D. and Sondhi, S. (2017). An Updated Checklist and Bibliography of the Birds of Uttarakhand. Published by Uttarakhand Forest Department, Dehradun.

Nameer, P.O. (1998). Checklist of Indian mammals. Kerala Forest Department, Thiruvananthapuram, Kerala, India.

Rawal, R.S. and Dhar, U. (2001). Protected area network in Indian Himalayan region: Need for recognizing values of low profile protected areas. Current Science, 81(2): 175-184.

Rawat, B., Negi, V.S., Mishra-Rawat, J. and Tewari, L.M. (2013). The Potential contribution of Wildlife Sanctuary to forest conservation: A case study from Binsar Wildlife Sanctuary. Journal of Mountain Science, 10(5): 854-865.

Rodgers, W.A. and Panwar, H.S. (1988). Planning a Wildlife Protected Area Network in India. Vol. I. The Report. Wildlife Institute of India, Dehradun.

Saxena, A.K. and Singh, J.S. (1982). A phytosociological analysis of woody species in forest communities of a part of Kumaun Himalaya. Vegetation, 50: 3-22.

Sharma, S., Rikhari, H.C. and Dhar, U. (1999). Designing of nature reserve to conserve a case study from Central Himalaya". Oecologia Montana, 8: 38-46.

Shahabuddin, G., Goswami, R. and Gupta, M. (2017). An annotated Checklist of the Birds of banjoak-chirpine forest in Kukaun, Uttarakhand. Indian Birds, 13(2): 29-36.

Singh, A. and Khushwah, S.P.S. (2011). Refining logistic Regression Models for Wildlife habitat suitability modeling- A Case study with Muntjak and Goral in the Central Himalayas. India Ecological Modelling, 222: 1354-1366.

Sultana, A. and Khan, J.A. (2000). Birds of oak forest in the Kumaun Himalaya, Uttar Pradesh, India. Forktail, 16: 131-146.

Tamta, P. (2017). Population dynamics and species diversity of Binsar Wildlife Sanctuary, Kumaun Himalayas, Uttarakhand, India. Ph. D. Thesis, Kumaun University, Naintal, pp. 343.

Uttarakhand Forest Department (2014). "Uttarakhand- A bird watching Heaven" in Uttarakhand Spring Bird Festival, 5-9 February, Sondhi, S. Eds. Uttarakhand.

Valdia, K.S. (1976). Kumaun Ki Khaniz Sampada: Kuch Sambhawanayen. Uttarakhand and Bhartiya, 1(2): 1-6.

ZSI, Editor- Director (2010). Fauna of Uttarakhand. State Fauna Series 18 (Part-I) Published by the Director, Zoological Survey of India, Kolkata. pp. 1-621.

Cite this chapter as: Arya, M.K., Badoni, A., Verma, A. and Ambika, T. (2021). An updated checklist of fauna of Binsar wildlife sanctuary of Uttarakhand (Western Himalaya), India. In: Biological Diversity: Current Status and Conservation Policies, Volume 1, Eds. Kumar., V., Kumar, S., Kamboj, N., Payum, T., Kumar, P. and Kumari, S. pp. 202-222, https:// doi.org/10.26832/aesa2021-bdcp-013 